\title{
Titan's ionosphere in the magnetosheath: Cassini RPWS results during the T32 flyby
}

\author{
P. Garnier ${ }^{1,2,3}$, J-E. Wahlund ${ }^{1}$, L. Rosenqvist ${ }^{1}$, R. Modolo ${ }^{1}$, K. Agren $^{1}$, N. Sergis ${ }^{4}$, P. Canu ${ }^{5}$, M. Andre ${ }^{1}$, \\ D. A. Gurnett ${ }^{6}$, W. S. Kurth ${ }^{6}$, S. M. Krimigis ${ }^{7}$, A. Coates ${ }^{8}$, M. Dougherty ${ }^{9}$, and J. H. Waite ${ }^{10}$ \\ ${ }^{1}$ Swedish Institute of Space Physics, P.O. Box 537, 75121 Uppsala, Sweden \\ ${ }^{2}$ Universite de Toulouse, UPS, CESR, 9 ave colonel Roche, 31028 Toulouse cedex 9, France \\ ${ }^{3}$ CNRS, UMR5187, 31028 Toulouse, France \\ ${ }^{4}$ Office for Space Research and Applications, Academy of Athens, Athens, Greece \\ ${ }^{5}$ Centre d'Etudes des Environnements Terrestre et Planetaires, Institut Pierre Simon Laplace, Velizy, France \\ ${ }^{6}$ Department of Physics and Astronomy, University of Iowa, Iowa City, IA 52242, USA \\ ${ }^{7}$ Applied Physics Laboratory, Johns Hopkins University, Laurel, MD, USA \\ ${ }^{8}$ Mullard Space Science Laboratory, University College London, Dorking, UK \\ ${ }^{9}$ Space and Atmospheric Physics group, Imperial College London, Prince Consort Road, London SW7 2AZ, UK \\ ${ }^{10}$ Space Science and Engineering Division, Southwest Research Inst., P.O. Drawer 28510, San Antonio, TX 78228-0510, \\ USA
}

Received: 11 May 2009 - Revised: 8 October 2009 - Accepted: 22 October 2009 - Published: 16 November 2009

\begin{abstract}
The Cassini mission has provided much information about the Titan environment, with numerous low altitude encounters with the moon being always inside the magnetosphere. The only encounter taking place outside the magnetopause, in the magnetosheath, occurred the 13 June 2007 (T32 flyby). This paper is dedicated to the analysis of the Radio and Plasma Wave investigation data during this specific encounter, in particular with the Langmuir probe, providing a detailed picture of the cold plasma environment and of Titan's ionosphere with these unique plasma conditions. The various pressure terms were also calculated during the flyby. The comparison with the T30 flyby, whose geometry was very similar to the T32 encounter but where Titan was immersed in the kronian magnetosphere, reveals that the evolution of the incident plasma has a significant influence on the structure of the ionosphere, with in particular a change of the exo-ionospheric shape. The electrical conductivities are given along the trajectory of the spacecraft and the discovery of a polar plasma cavity is reported.
\end{abstract}

Keywords. Ionosphere (Ionospheric irregularities; Planetary ionospheres) - Magnetospheric physics (Magnetosheath)

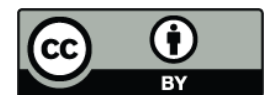

Correspondence to: P. Garnier (philippe.garnier@cesr.fr)

\section{Introduction}

The Cassini mission has substantially progressed our knowledge of the Saturnian environment since the 1 July 2004, in particular regarding the largest satellite, Titan. Its dense molecular nitrogen atmosphere (Waite et al., 2005) is not protected by an intrinsic magnetic field, thus interacting directly with the Saturnian magnetosphere (Backes et al., 2005).

The ionization of the Titan's atmosphere, mainly due to solar UV photoionization and electron impact ionization, leads to a thick and extended ionosphere, analyzed in detail during the first Cassini flyby Ta (Wahlund et al., 2005). The numerous Titan flybys (the 50th occurring in February 2009) showed a complex and dynamic view of Titan's ionosphere, giving a better understanding of its composition (Cravens et al., 2006, 2009) as well as its various sources and losses (Ȧgren et al., 2007; Modolo et al., 2007a,b), and showing a mostly solar-driven structure (Ȧgren et al., 2009). Furthermore, the ion chemistry taking place in the ionosphere appeared more complex than foreseen by the previous modeling studies (Keller et al., 1998; Vuitton et al., 2006), with in particular the discovery of heavy negative ions (up to $10000 \mathrm{amu} ; 1 \mathrm{amu} \sim 1.66 \times 10^{-27} \mathrm{~kg}$ ) in the lower ionosphere (Coates et al., 2007a).

Titan is located in the outer part of the Saturnian system $\left(\sim 20 R_{S}, R_{S}=60268 \mathrm{~km}\right.$ Saturn radius), and is most of the time inside the magnetosphere, even at the subsolar point (Lundberg et al., 2005): the mean bi-modal magnetopause stand-off distance is indeed $\sim 22-27 R_{S}$ (Achilleos

Published by Copernicus Publications on behalf of the European Geosciences Union. 
et al., 2008). However, a strong solar wind pressure may induce the presence of the moon in the magnetosheath, or even in the solar wind.

T32, which occurred on 13 June 2007, is the first flyby reported where Titan encountered a non-magnetospheric plasma. Titan was indeed located in the magnetosheath region, embedded in a shocked solar wind plasma, very close to the magnetopause. During this pass, the MAG experiment (magnetic field data) exhibited the presence of "fossil" field lines (Bertucci et al., 2008), with a magnetospheric-like configuration in the atmosphere, very different from the magnetosheath magnetic field configuration observed at higher altitudes. The interpretation of the observations is complicated for this flyby, with a combination of different plasmas characterizing several regions (magnetosphere, magnetosheath, Titan ionosphere) crossed by Cassini in less than one hour.

In this paper, we analyze in detail the Cassini RPWS (Langmuir probe in particular) observations (Gurnett et al., 2004) during the T32 flyby, in order to study the unknown response of the ionosphere to a magnetosheath plasma environment. A comparison is shown with a previous encounter, the T30 flyby (12 May 2007), whose geometry is very similar to the T32 pass but where Titan was inside the magnetosphere. We also present electrical conductivities calculated in the extended ionosphere, and we finally report the discovery of a polar plasma cavity whose formation process is discussed.

\section{The RPWS Langmuir probe data}

The Langmuir probe (also referred to as LP in the paper) is one of the sensors of the Cassini Radio and Plasma Waves experiment (Gurnett et al., 2004) and is dedicated to the measurement of the in situ parameters of cold and dense plasmas, such as the electron density and temperature, the ion flow and total energy (ram and thermal energy), as well as the spacecraft potential. It can thus perform a detailed analysis of Titan's ionosphere.

The probe itself consists of a titanium sphere mounted on a boom. The bias voltage of the probe is varied every $24 \mathrm{~s}$ between $-4 \mathrm{~V}$ and $+4 \mathrm{~V}$ during a Titan flyby occurring at a closest approach below $1200 \mathrm{~km}$. The analysis of the currentvoltage curve of the sweeps, using the Orbital Motion Limited (OML) theory (Mott-Smith and Langmuir, 1926), allows to estimate several electron and ion parameters (Fahleson et al., 1974).

The electron density $n_{e}$ will be derived here through two independent methods, using the Langmuir probe and the upper hybrid frequency measured by the RPWS electric antennas:

- first, the current measured during the sweeps with positive voltages can provide, for each electron population determined, the density $n_{e}$ as well as the temperature $T_{e}$ and the floating potential $U_{\text {float }}$ (then leading to the true spacecraft potential $U_{s c}$ after a correction for the photoelectrons cloud and the size of the boom).

- then, between the sweeps, the probe has a continuous mode, with the current being sampled at a constant bias voltage $(+4 \mathrm{~V}$ for this flyby) at a frequency of $20 \mathrm{~Hz}$ : the continuous density $n_{e_{20}}$ is provided, assuming the potential and the temperature are constant between two sweeps.

- these two first methods can only be used in a rather dense plasma $\left(n_{e}>5 \mathrm{~cm}^{-3}\right)$, but not in the thin outer magnetospheric plasma $\left(n_{e}<1 \mathrm{~cm}^{-3}\right)$ where the spacecraft photoelectrons dominate (except in eclipse). In this case, the floating potential derived from the LP sweeps analysis is used as a proxy to estimate the electron number density (M. Morooka, personal communication; Modolo et al., 2007a).

- finally, the electron density can also be obtained from RPWS waves spectrograms in dense plasmas $\left(n_{e}>\right.$ $1 \mathrm{~cm}^{-3}$ ). Resonant emissions occur indeed at the upper hybrid frequency $f_{u h}=\sqrt{f_{g e}^{2}+f_{p e}^{2}}$ (with $f_{g e}$ and $f_{p e}$ being respectively the electron gyro-frequency and plasma frequency) which leads, via the knowledge of the magnetic field, to the electron density. The cyclotron frequency is usually much smaller than the plasma frequency, thus the upper hybrid frequency is mostly the plasma frequency.

The other part of the Langmuir probe sweeps, with negative voltages, measures both the ion and photoelectron currents. The photoelectrons act as a useful constant noise, inducing a total current above the background noise, which then leads to the ion current. This current is proportional to the ion flux $n_{i} v_{i}$, and the slope of the curve provides the total ion energy $W_{i, \text { eff }}$ (kinetic + thermal energies).

- the ion ram velocity $v_{i, \text { ram }}$ can be estimated with two independent methods. It can be derived from the ion flux, assuming the charge neutrality $\left(n_{e}=n_{i}\right)$, or from the total energy $W_{i, \text { eff }}$ : in the ionosphere, the thermal energy is negligible compared with the ram energy (the spacecraft velocity $v_{s c}$ is of $\sim 6 \mathrm{~km} / \mathrm{s}$ during the Titan encounters), which leads to $v_{i \text {,ram }}$ if an assumption is made for the ion mass $m_{i}$.

- the ion density can also be estimated, independently from the electrons, with assuming a ram velocity (i.e. the spacecraft velocity $v_{s c}$ in the cold ionosphere) and using the ion flux measured $n_{i} v_{i}$.

- finally, the last parameter derived from the Langmuir probe, with a reduced precision, is the ion mass $m_{i}$ in the ionosphere: with the knowledge of the total energy, instead of assuming an ion mass to obtain the ion ram velocity, we assume the ram velocity (previously given 
by the ion flux with the charge neutrality assumption) to estimate the averaged value of the ions mass.

Furthermore, we use here a new method to estimate the electron temperature in the interface region between $\mathrm{Ti}$ tan's ionosphere and the magnetospheric or magnetosheath plasma, where the RPWS Langmuir probe or the CAPS electron spectrometer (ELS) reach their limitation. Combining the wideband receiver data (WBR) with the $20 \mathrm{~Hz}$ Langmuir probe (LP) data we are indeed able to provide electron temperature at high temporal resolution. The continuous LP mode (20 samples/s) samples electron current, $I_{e}$, at a given potential $V_{p}$. The electron current is defined as $I_{e}=I_{e_{0}} \exp \left(-\chi_{e}\right)$ or $I_{e}=I_{e_{0}}\left(1-\chi_{e}\right)$ when $V_{p}+V_{f}$ is positive or negative respectively, meaning that the probe attracts or repels electrons depending on the potential. $V_{f}$ is the floating potential estimated from the Langmuir probe sweeps, $I_{e_{0}} \propto n_{e} \sqrt{T_{e}}$ and $\chi_{e} \propto\left(V_{p}+V_{f}\right) / T_{e}$. Assuming that the floating potential deduced from the LP sweeps varies linearly between two sweeps, separated by $24 \mathrm{~s}, V_{f}$ can be extrapolated to the time resolution of the WBR. The electron number density $n_{e}$ can be estimated from the upper hybrid resonant emission, when a clear signature is identified, on the wideband receiver data every $2.4 \mathrm{~s}$. Using the $I_{e}$ values from $20 \mathrm{~Hz}$ LP data, $n_{e}$ from WBR and extrapolated $V_{f}$ from LP sweeps, one can provide electron temperature values on the time resolution of $\mathrm{WBR}$. There is no limitation on $T_{e}$ values, it can vary from few $\mathrm{eV}$ to few hundreds of $\mathrm{eV}$, whereas the classic (ionospheric) method hardly exceeds $T_{e} \sim 5 \mathrm{eV}$ (the temperature of particles, when expressed in $\mathrm{eV}$ in our paper, actually refers to the thermal energy $k_{B} * T$ with $k_{B}$ the Boltzmann constant). The frequency range of the WBR is $1 \mathrm{kHz}$ to $80 \mathrm{kHz}$, providing a limitation on the electron number density (theoretically $n_{e}$ needs to be smaller than $80.0 \mathrm{~cm}^{-3}$ and larger than $0.01 \mathrm{~cm}^{-3}$ ).

\section{Analysis of the T32 flyby}

\subsection{The plasma environment}

The T32 flyby occurred on 13 June 2007, during the outbound part of the Cassini's revolution 46, at a Saturn local time of $\sim 13.6 \mathrm{~h}$, with a closest approach at 17:46 for a minimum altitude of about $975 \mathrm{~km}$. As can be seen in Fig. 3, where the T32 flyby geometry is shown, the spacecraft flew from the inner magnetosphere towards the magnetosheath, passing over the northern pole of the moon.

The Fig. 1 gives an overview of the Langmuir probe results during the flyby. The seven panels give various parameters as a function of the time (UT), the distance of the spacecraft from Titan (in Titan radii $R_{T}=2575 \mathrm{~km}$ ) as well as the local time (LT) and latitude (LAT) with respect to the moon.

We need to note first the existence of an eclipse period for the spacecraft during most of the inbound leg of the flyby (between 17:00 and 17:40-17:45 UT). This eclipse period for the probe, due to the presence of Titan between Cassini and the Sun, is determined either from the Cassini trajectory with respect to the moon or from the LP observations by the lack of contribution of photoelectron currents on the ion current part of the sweep data (Fig. 1 panel a).

This induces a major constraint for the analysis of the ambient ions, since no photoelectron current will be created at the surface of the probe, preventing the visualization of the ion current which keeps indeed hidden by the background noise. However, the detailed fitting of the individual Langmuir probe sweeps reveals the presence of a small solar flux, which is probably due to the light scattering through the dense Titan atmosphere.

The second constraint induced by the eclipse period is the possibly large negative values for the spacecraft's potential, itself causing larger uncertainties in fitting the probe sweeps. As a consequence, we focused, in the inbound leg, on the dense region, where the upper hybrid emissions may be used as a reference for the electron density. The electron temperature is difficult to estimate in a thin plasma, since LP sensors are well designed for dense and cold $\left(n_{e}>1 \mathrm{~cm}^{-3}\right.$, $T_{e}<5 \mathrm{eV}$ ) plasma regions.

The inbound leg mainly shows a progressive increase in the electron density, from $\sim 0.3 \mathrm{~cm}^{-3}$ at $17: 25$ up to a first significant peak at $\sim 10 \mathrm{~cm}^{-3}$ around 17:32 and a second similar peak $5 \mathrm{~min}$ later (corresponding to an altitude of about $2300 \mathrm{~km}$ ). The electron temperature is highly variable (around $10 \mathrm{eV}$ ) and we have no ion data in that part. The Cassini spacecraft then enters the deep ionosphere (around 17:40-17:54) which will be discussed in Sect. 4.1.

An important question is the nature of the inbound rather dense and cold plasma, observed from around 17:30: is it of magnetospheric/magnetosheath/ionospheric origin? Bertucci et al. (2008) studied the T32 flyby, focusing on the magnetic field topology and using various Cassini data. They showed that Cassini crossed the magnetopause around 17:20-17:30, leaving the magnetosphere and entering the magnetosheath, where Titan was lying during a period estimated between $20 \mathrm{~min}$ and $3 \mathrm{~h}$ at the time of the closest approach.

The magnetosheath plasma is expected to be colder and denser than the magnetospheric plasma: $\sim 50 \mathrm{eV}$ and $\sim 1-$ $10 \mathrm{~cm}^{-3}$ (Bridge et al., 1982; Richardson, 1987) instead of $\sim 200 \mathrm{eV}$ and $\sim 0.1 \mathrm{~cm}^{-3}$ (Wahlund et al., 2005). Thus, the cold and dense plasma observed (after 17:30) could be either from the magnetosheath or the extended ionosphere, the second case inducing a colder temperature (typically below $10 \mathrm{eV}$ ). As a consequence, the Langmuir probe results (Fig. 1 panel c)) are in agreement with an ionospheric origin - at least partly - for the cold and dense plasma observed in the inbound leg of the flyby, rather than a pure magnetosheath origin.

Moreover, Coates et al. (2007b) and Ma et al. (2009) reported the cold plasma parameters given by the CAPS-ELS instrument during the flyby, which provides better estimates 


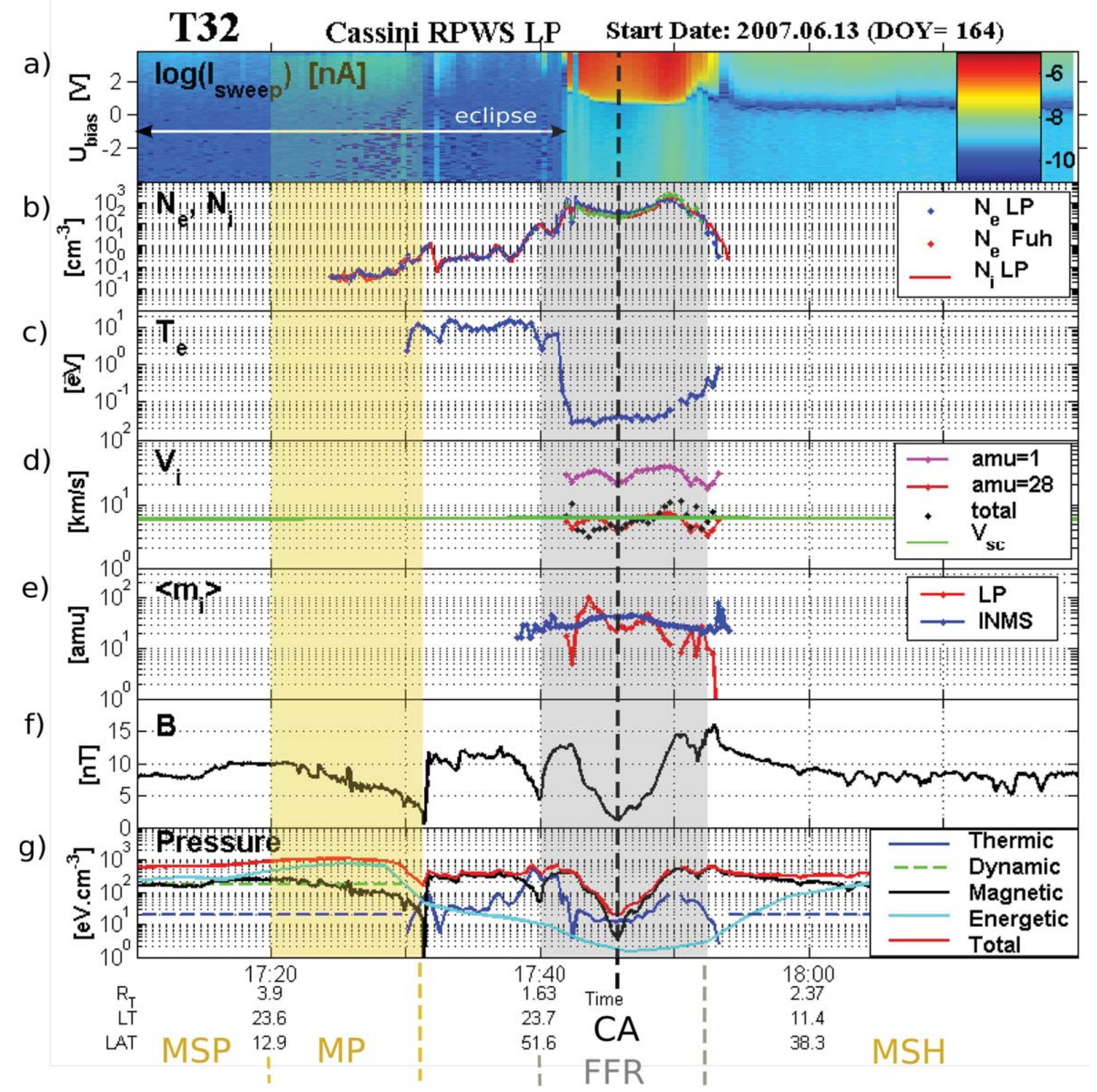

Fig. 1. Cassini Langmuir probe results during the T32 Titan flyby, as a function of time: (a) raw Langmuir probe data, with the current (nA) color coded, as a function of time and bias voltage $(V)$; (b) Langmuir probe electron (blue) and ion (green) densities $\left(\mathrm{cm}^{-3}\right)$, and upper hybrid derived electron densities (red); (c) electron temperature $(\mathrm{eV}) ;(\mathbf{d})$ spacecraft velocity (green, $\mathrm{km} / \mathrm{s})$; ion ram velocity $(\mathrm{km} / \mathrm{s})$ : from the ion flux and assuming charge neutrality (black), or from the total ion energy and assuming an ion mass (1 amu in magenta, 28 amu in red); (e) averaged ion mass (amu), derived from the Langmuir probe (red) and the INMS experiment (blue); (f) total magnetic field (nT) measured by the MAG experiment; $(\mathbf{g})$ pressures $\left(\mathrm{eV} \mathrm{cm}^{-3}\right)$ : electron thermal (blue; from Langmuir probe - continuous - or estimated - dashed -), dynamic (estimated, green), magnetic (MAG experiment, black), energetic (MIMI/CHEMS instrument, cyan) and total (red). Cassini crossed the magnetopause (MP) around the yellow area, from the magnetosphere (MSP) to the magnetosheath (MSH), and the fossil fields region (FFR) discovered by Bertucci et al. (2008) is shown by the gray area. See text for more details. 
than the Langmuir probe in the thin plasma regions. The electron densities are, in particular, much lower in the outbound pure magnetosheath region than in the inbound dense region, and the electron temperature given by CAPS shows clear transitions: from the magnetospheric plasma towards the magnetosheath plasma (around 17:20-17:30) with a temperature decreasing from $\sim 100-200 \mathrm{eV}$ down to $\sim 40-50 \mathrm{eV}$, and a second one towards the ionospheric plasma (just before 17:30) with a sudden temperature decrease (Ma et al., 2009).

The outbound leg of the flyby shows a sharper transition between the cold and dense ionospheric plasma and the ambient thinner magnetosheath plasma (around 17:54). The spacecraft is not in eclipse during this period, which prevents us from providing the electron temperature since the photoelectrons may dominate in such a thin plasma. Actually, it appears that the probe is at the border between the so-called "thin" and "dense" regimes. Assuming a thin plasma, dominated by photoelectrons, leads to electron densities of $\sim 0.2-$ $0.3 \mathrm{~cm}^{-3}$, whereas assuming a dense regime, without dominant photoelectrons, provides densities around $5-10 \mathrm{~cm}^{-3}$. The CAPS experiment, which is expected to be more accurate in these regions, found values of $\sim 0.4-0.6 \mathrm{~cm}^{-3}$ and a temperature $T_{e} \sim 40 \mathrm{eV}$ (see Fig. 2 in Ma et al., 2009). Moreover, the analysis of the RPWS data suggests the existence of a noisy upper hybrid line, very close to the limit of detection, indicating electron densities in the range of $0.3-1$ or $2 \mathrm{~cm}^{-3}$, which is in agreement with the CAPS derived values and the expectation of densities slightly larger than in the magnetosphere.

\subsection{The pressure environment}

The panel (g) in Fig. 1 shows a pressure analysis during the pass, with the main pressures: electron thermal (blue), dynamic (green), magnetic (black) and energetic (cyan). The electron thermic component is defined by $p_{t h_{e}}=n_{e} k_{B} T_{e}$. We assume $T_{e}=200$ or $50 \mathrm{eV}$ and $n_{e}=0.1$ or $0.5 \mathrm{~cm}^{-3}$, respectively, in the magnetosphere or the magnetosheath (dashed blue curve in Fig. 1). The ion temperature is much larger (Hartle et al., 2006a, b) in the magnetosphere, where the suprathermal pressure (described below) thus contains most of the ion contribution. In the ionosphere, we also keep only the electron pressure (continuous blue), with $n_{e}$ and $T_{e}$ directly measured, with the ion temperature being probably close to the neutral temperature $-\sim 150 \mathrm{~K}$ or $\sim 0.013 \mathrm{eV}-$ in the lower atmosphere, due to cooling by multiple collisions with neutrals, and then increasing towards $T_{e}$ in the upper atmosphere (see Roboz and Nagy, 1994, and Sect. 4.1 for more details).

The dynamic pressure $\left(p_{\text {dyn }}=\left(n_{\mathrm{H}^{+}} m_{\mathrm{H}^{+}}+n_{\mathrm{O}^{+}} m_{\mathrm{O}^{+}}\right) v_{i}^{2}\right)$ is only estimated in the magnetospheric region - with $n_{\mathrm{O}^{+}}=$ $2 * n_{\mathrm{H}^{+}}=2 / 3 * n_{e}=0.067 \mathrm{~cm}^{-3}, m_{\mathrm{O}^{+}}=16 * m_{H^{+}}=16 \mathrm{amu}$ (protons and oxygen ions) and $v_{i}=120 \mathrm{~km} / \mathrm{s}$ (Hartle et al., 2006a, b; Neubauer et al., 1984) - since the magnetosheath velocity is unknown. We may add that Cassini results in- dicate a smaller ratio $n_{\mathrm{O}^{+}} / n_{\mathrm{H}^{+}}$(down to $1 / 4$; Crary et al., unpublished manuscript), which would much decrease (by a factor 2 or 3) our dynamic pressure estimate based on the Voyager conditions. The magnetic field pressure is derived from the Cassini MAG data (Dougherty et al., 2004): $p_{B}=\frac{B^{2}}{2 \mu_{0}}(B$ is the magnetic field in $\mathrm{nT}$ shown in panel (f) of Fig. 1 and $\mu_{0}$ the magnetic permeability of vacuum).

The last pressure term is given by the energetic particles, deduced from the MIMI CHEMS data (Krimigis et al., 2004) using the method developed by Sergis et al. (2007). This suprathermal pressure corresponds to both $\mathrm{H}^{+}$and $\mathrm{O}^{+}$(major plasma components) for energies above $3 \mathrm{keV}$.

The inbound magnetospheric leg of the encounter is dominated by the suprathermal and magnetic pressures, the first one being known as, on averaged, the major pressure contribution in the outer magnetosphere (Sergis et al., 2007, 2009). The estimated dynamic pressure is of the same order of the magnetic term, whereas the electron thermic pressure is smaller by a factor 10 . The magnetopause crossing is followed by the Titan interaction region, with an increasing thermal pressure and a magnetic field pile-up on the edges of the Titan's atmosphere. The thermal pressure becomes the dominant component only in the deep ionosphere, where the Saturnian magnetic field vanishes (below $1000 \mathrm{~km}$ ).

The magnetic pile-up may attenuate the penetration of ambient electrons $\left(\Delta p_{B_{\max }} \sim 300-400 \mathrm{eV} \mathrm{cm}^{-3}\right.$ in outbound, to be compared with $p_{e} \sim 20 \mathrm{eV} \mathrm{cm}^{-3}$ ), preventing them from ionizing efficiently the deep atmosphere, even if Gan et al. (1992) showed that magnetospheric electrons can still partially enter Titan's atmosphere in the case of highly draped magnetic field lines. It may have an influence for the discrepancy, during several flybys, between the observed nightside electron densities measured and modeled, using the incident CAPS electron fluxes (Ägren et al., 2007; Cravens et al., 2009).

The outbound magnetosheath region is most probably dominated by the thermal pressure of the shocked solar wind particles (in particular protons and helium ions), followed by the magnetic and suprathermal pressures which are similar. The dynamic pressure is unknown, since we lack information about the magnetosheath velocity, which is probably highly variable, depending on both the solar wind velocity and the region studied.

\section{The Titan's ionosphere}

\subsection{The ionosphere during the T32 flyby}

The Fig. 2 provides an altitude zoom (900-2400 km) of the Langmuir probe parameters, for both electrons and ions as well as the inbound and outbound legs of the flyby.

There is a rough symmetry between the two legs of the flyby, with similar altitude profiles for the various parameters (except for the ions, since the eclipse period prevented 

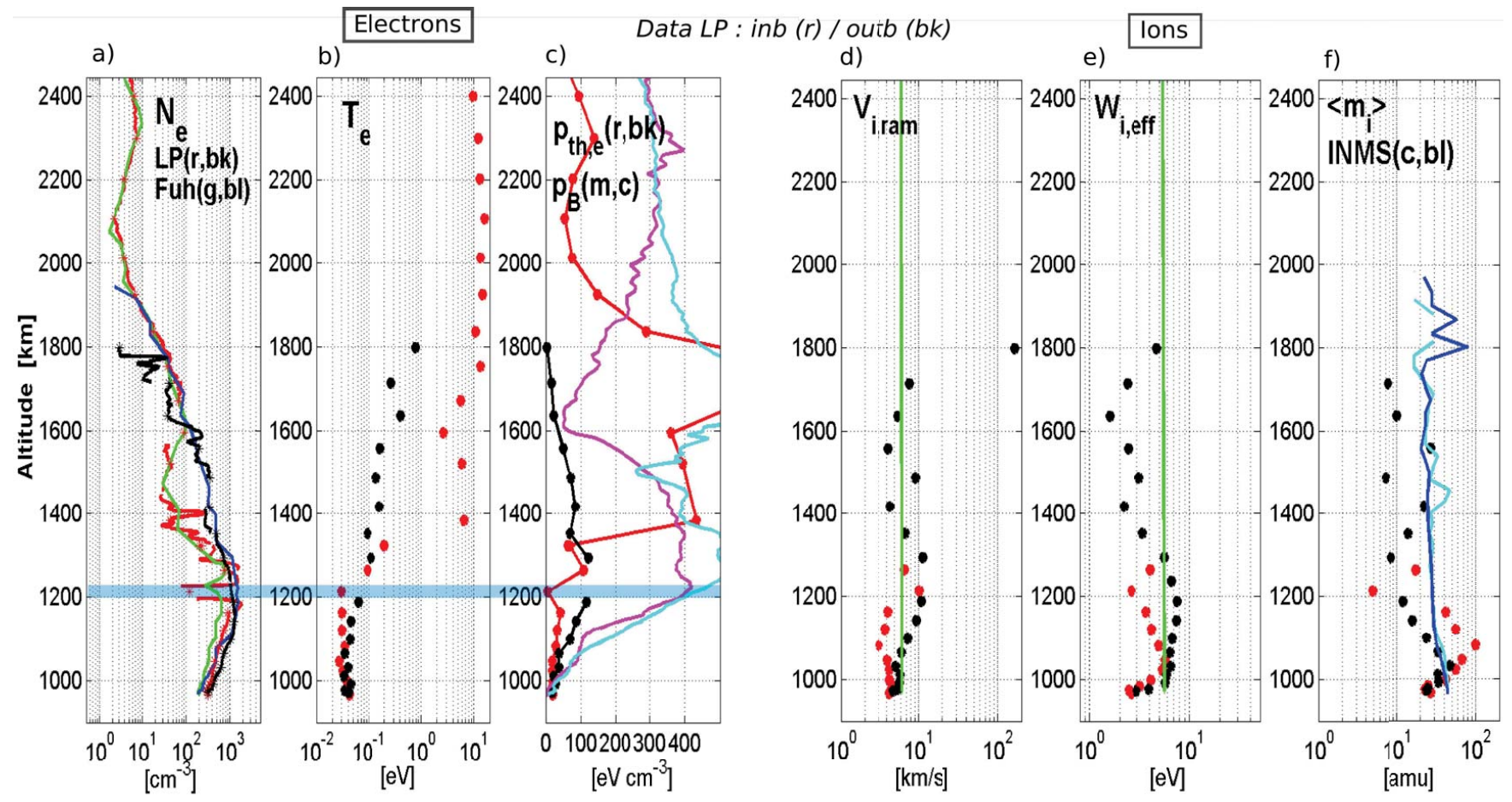

Fig. 2. Altitude $(\mathrm{km})$ profiles of electron and ion parameters in Titan's ionosphere: (a) electron density $\left(\mathrm{cm}^{-3}\right)$ derived from the Langmuir probe (inbound part of the flyby in red, outbound in black) and the upper hybrid line (inbound in green, outbound in cyan); (b) electron temperature (eV; inbound in red, outbound in black); (c) pressures $\left(\mathrm{eV} \mathrm{cm}^{-3}\right)$ : electron thermal (inbound in red, outbound in black) and magnetic pressure (inbound in magenta, outbound in cyan); (d) ion ram velocity ( $\mathrm{km} / \mathrm{s}$; inbound in red, outbound in black) and spacecraft velocity $(\mathrm{km} / \mathrm{s}$; green); (e) total (ram + thermal) ion energy $(\mathrm{eV})$ and spacecraft's kinetic energy (eV; green); (f) ion averaged mass (amu) derived from the Langmuir probe (inbound in red, outbound in black) and the INMS experiment (inbound in cyan, outbound in blue).

us from providing ion parameters during a large part of the inbound leg). However, a closer look reveals larger electron temperatures in the inbound upper ionosphere (around 1400$1800 \mathrm{~km}$ altitude), inducing a larger thermal electron pressure in the nightside part of the ionosphere. The difference may come from the different ionization sources between the two legs (electron impact in inbound, photoionization in outbound), but the T5 flyby revealed an opposite situation, with larger temperatures in the dayside ionosphere (Ägren et al., 2007). The variability of the magnetic field configuration, observed during the pass (Bertucci et al., 2008), is probably a better explanation, since its topology has a large influence on the electron temperature (Galand et al., 2006).

Moreover, we observe a larger variability for the electron density in the inbound leg, which may be due to the variability of both the plasma and the magnetic field induced by the crossing of the magnetopause. The peak altitude also slightly changes, from $1200-1250 \mathrm{~km}$ in inbound to $1175 \pm 25 \mathrm{~km}$ in outbound (or respectively from $\sim 1175$ to $\sim 1145 \mathrm{~km}$ if we consider only the sweep points). Such a decrease was expected, since the solar zenith angle is larger in inbound $\left(130^{\circ} / 140^{\circ}\right.$ compared to $\left.85^{\circ} / 90^{\circ}\right)$, inducing a higher peak altitude (Ȧgren et al., 2009). The maximum electron densi- ties observed during the probe sweeps are respectively $\sim 900$ and $1300 \mathrm{~cm}^{-3}$, but the inbound continuous data $\left(n_{e_{20}}\right)$, assuming a constant temperature between the sweeps) show a highly variable plasma density, with values up to $1900 \mathrm{~cm}^{-3}$. This would lead to an unexpected larger density in the nightside ionosphere where the solar flux cannot efficiently ionize the atmosphere.

The magnetic pressure is large compared to the thermal pressure even at low altitudes, with a ionopause - defined by $p_{B}=p_{t h}-$ around $1000 \mathrm{~km}$ altitude (for $T_{i} \sim T_{n} ;$ or $1050 \mathrm{~km}$ assuming $T_{i}=T_{e}$ in the lower ionosphere), well below the ionospheric peak. Such a dominant magnetic pressure may lead to significant electrodynamics (see Sect. 6), and underlines the importance of the collisions to prevent the ionosphere from being dragged by the draping Saturn's magnetic field. The ion ram velocity profile in Fig. 2 reveals relative velocities (with respect to the spacecraft velocity) up to a few $\mathrm{km} / \mathrm{s}$, which may correspond to ion flows (and thus electrodynamic processes) but also neutral winds. However, MüllerWodarg et al. $(2006,2008)$ inferred horizontal neutral winds, based on the Ion Neutral Mass Spectrometer data (Waite et al., 2004), up to only $300 \mathrm{~m} / \mathrm{s}$. 
The total ion energy $W_{i \text {,eff }}$ (kinetic + thermal energies; Fig. 2 panel e), is assumed to be dominated by the kinetic energy, which allows to estimate the expected ion ram velocities for various ion species (panel d, Fig. 1). Thus the ion ram velocity is in agreement with heavy species ions (28 amu) in the ionosphere, and with protons at high altitudes (a common feature for all the flybys). The other possibility is to infer the averaged ion mass (Fig. 2 panel f) from this total energy, knowing the ion ram velocity ( $v_{i, \text { ram }}$ in panel d), which reveals a large variability during the flyby, with increasing values near closest approach. The derived values are in reasonable agreement with the INMS results, which show stable values roughly around the expected $28 \mathrm{amu}$ of the $\mathrm{HCNH}^{+}$dominant ion species. Moreover, the Langmuir probe takes into account all ion species, whereas these INMS results do not consider species with masses above $100 \mathrm{amu}$ : such heavy ions are present at low altitudes with masses up to $\sim 40000 \mathrm{amu}$ (Coates et al., 2007a; Waite et al., 2007) and significant amounts, thus explaining the large discrepancies often observed near the Cassini's closest approach between the RPWS and INMS results (with a factor 3-4 during T32 inbound at $1100 \mathrm{~km}$ altitude).

The ion temperature is not provided directly by the Langmuir probe, but rough estimates may be given, either using the ionospheric scale height or calculating Eq. (1) below. The scale height of the ionosphere during T32, given by $H_{i}=\frac{k_{B}\left(T_{i}+T_{e}\right)}{g\left(m_{i}+m_{e}\right)}\left(g\right.$ is the Titan gravity and $m_{e}$ the electron mass), is of the order of a few hundreds of $\mathrm{km}-\sim 200 \mathrm{~km}$ on average -, with a large variability of the density gradient which prevents from providing an altitude profile of the ion temperature.

In the collisional ionosphere, below the exobase $(\sim 1425 \mathrm{~km})$, the temperature of the dominant ion species $\left(\mathrm{HCNH}^{+}\right)$may be derived from the energy equation through (Schunk and Nagy, 2000; Blelly and Alcayde, 2007):

$$
T_{i}=\frac{v_{i n}}{v_{i n}+v_{i e}}\left(T_{n}+\frac{m_{n}}{3 k_{B}}\left(u_{i}-u_{n}\right)^{2}\right)+\frac{v_{i e}}{v_{i n}+v_{i e}} T_{e}
$$

with the neutrals temperature $T_{n}=150 \mathrm{~K}$ (Waite et al., 2005) and mass $m_{n}=28 \mathrm{amu}\left(\mathrm{N}_{2}\right)$, the ion-neutral and ion-electron collision frequencies $v_{i n}$ and $v_{i e}$ (given by Kelley et al., 1989; Banks and Kockarts, 1979), and the ions and neutrals velocities $u_{i}$ and $u_{n}$; we use our exosphere model (Garnier et al., 2007) combined with the revised version of the atmospheric model of Toublanc et al. (1995) for the neutrals densities.

If we consider the ions at rest with respect to the neutrals $\left(u_{i}=u_{n}\right), T_{i}$ is close to $T_{n}$ as long as the thermalizing collisions between ions and neutrals are dominant $\left(\alpha=\frac{\nu_{i n}}{v_{i e}} \gg 1\right)$ and then reaches $T_{e}$ when the ion-electron collisions dominate $(\alpha \ll 1)$ : this transition occurs above the exobase, around $2000-3000 \mathrm{~km}$ altitude, mainly due to the decreasing exospheric neutral densities. However, if we consider the ion ram velocities derived by the Langmuir probe by stating $\left|u_{i}-u_{n}\right|=\left|v_{I_{0}}-v_{s c}\right|=\delta v$ (neutrals at rest), the ion temperature may increase very much, with even larger values than $T_{e}$ in the collisionnal ionosphere: for example, a $\delta v$ of $0.5 \mathrm{~km} / \mathrm{s}$ leads to an increase of $\sim 280 \mathrm{~K}$ for $T_{i}$.

The cold and dense plasma region located above the exobase may be called the exo-ionosphere (Wahlund et al., 2005) and, given the complex plasma environment near the magnetopause (Sect. 3.1), is rather extended in the inbound leg of T32 (up to a distance from Titan of $\sim 2.5 \pm 0.2 R_{T}$ ). This region is characterized by decreasing densities with altitude, increasing ion ram velocities, larger electron temperature values than in the collisional ionosphere, and a larger magnetic pressure than the thermal pressure. The magnetospheric/magnetosheath plasma is the key driver for the dynamics of this transport dominated region, with matter picked-up downstream and a significant mass loading by the ionosphere which slows down the ambient plasma. Unfortunately, we do not have the ion ram velocity profile during the whole dataset, which prevents us from determining the location of the Mass Loading Boundary.

Actually, the altitude of the ion exobase, where the mean free path of the ions $\lambda_{i}$ is equal to the density scale height $H_{i}$, is slightly higher than the neutral exobase (Lemaire and Scherer, 1974) and depends on each species. The knowledge of the collision frequencies (previously given) and of the density scale height (calculated from the Langmuir probe data), combined with the ion temperature, leads to a rough estimation of this critical level for the main ion species $\mathrm{HCNH}^{+}$. Assuming $T_{i}=T_{n}$ leads to an ion exobase at $\sim 1675 / 1585 \mathrm{~km}$ in inbound/outbound (with $\lambda_{i} \sim H_{i} / 10$ at the neutral exobase), and considering the probe ram velocity in Eq. (1) leads to slightly lower altitudes: 1475-1650 and $1525 \mathrm{~km}$, respectively, in inbound and outbound, due to an increased mean free path. Thus, the ion exobase is on average $\sim 100 / 200 \mathrm{~km}$ higher than the neutral exobase during the T32 encounter.

\section{A comparison with the T30 flyby}

The Titan flyby T30 (12 May 2007) occurred during the outbound leg of the Cassini's revolution 45, with a geometry very similar to the flyby T32, with respect to both Saturn and Titan. The Saturn local time was indeed about $13.6 \mathrm{~h}$ (/13.6 h also for T32), for a closest approach altitude around $949 \mathrm{~km}(/ 959 \mathrm{~km})$ at 20:10. The spacecraft, whose trajectory is shown in Fig. 3 for both flybys, flew over the northern pole of the moon with an increasing latitude, from the nightside (eclipse) to the dayside. The solar zenith angle was slightly larger (by $\sim 15^{\circ}$ ) during T30 than during T32, with values of $\sim 140 / 100^{\circ}$ at the inbound/outbound ionospheric peaks. However, on the contrary to the flyby T32, T30 took place well inside the magnetosphere, due to a reduced solar wind pressure at that period. These characteristics thus make this encounter a very good opportunity to study the influence of the magnetosheath configuration on the Titan's ionospheric structure and dynamics, in particular in the nightside 


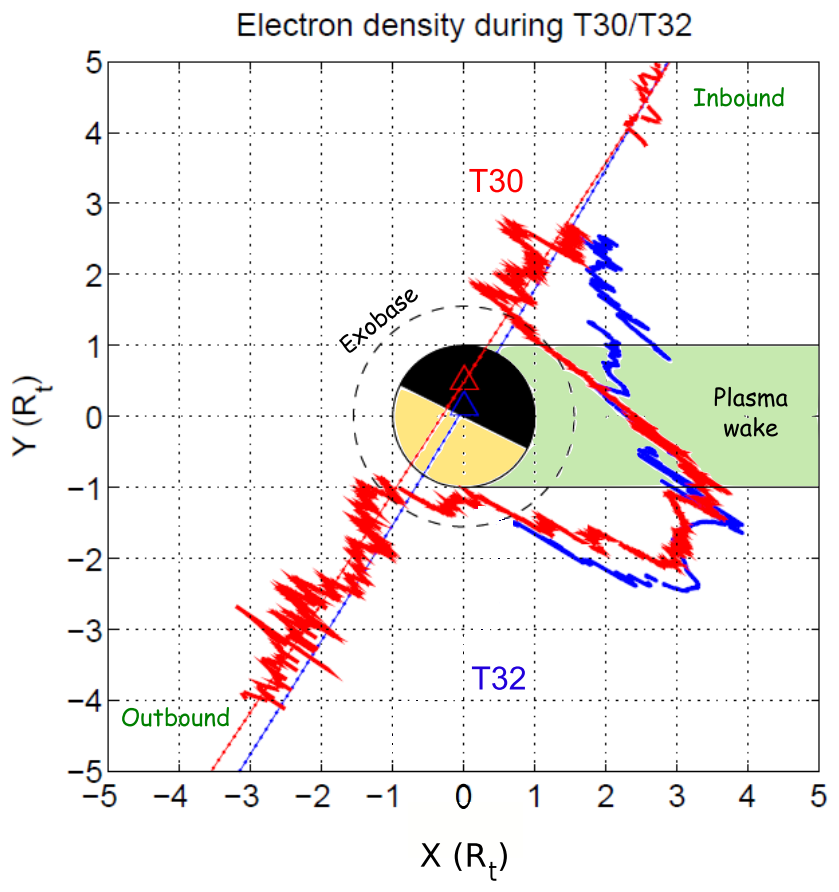

Fig. 3. Comparison between the T30 (red) and T32 (blue) Titan flybys. The Cassini trajectory (dashed lines) is given in the corotational frame ( $\boldsymbol{X}$ is the ideal corotation velocity, $\boldsymbol{Y}$ is the Saturn's direction and $\boldsymbol{Z}$ completes the orthonormal frame). The dayside and nightside parts of Titan are shown (yellow and black hemispheres), as well as its exobase (dashed black) and ideal plasma wake (green area, in the magnetospheric configuration). The electron density $\left(n_{e_{20}}\right)$ is superimposed along the Cassini trajectory for both T30 and T32 (red and blue lines).

region, where the electron impact ionization is the main energy source for the ionosphere.

The different characteristics between magnetosheath and magnetospheric electron spectra (see Sect. 3.1) should induce several differences in the nightside region:

- the lower energy of the magnetosheath electrons theoretically leads to a higher altitude for the ionization peak,

- the magnetosheath electron flux is globally increased (due to a larger density), which should increase the ionization rates and thus the ionospheric densities,

- the ram direction of the plasma may be very different, inducing a possible reconfiguration of the ionospheric shape

We have performed a detailed analysis of the T30 encounter Langmuir probe data, with the same method as for T32. The Fig. 3 provides the electron density $n_{e}$ along the spacecraft trajectory for both flybys. The first remarkable feature is the very different densities in the inbound leg of both encounters: during T30, there is no dense region similar to the T32 configuration (identified as mostly ionospheric in Sect. 3.1), with a pure magnetospheric plasma $\left(n_{e} \sim 0.1 \mathrm{~cm}^{-3}\right)$. Thus, a ram direction like the magnetospheric (ideal) corotation should not lead to a ionospheric plasma region in the inbound Saturn's facing side (the accelerating corotation electric field is all the more in the opposite direction). As a consequence, the T32 results are in agreement with a magnetosheath ram direction similar to the solar wind direction, inducing the observation of significant ionospheric plasma dragged downstream. It might also be in agreement with a magnetospheric corotation direction very different from the ideal direction (Szego et al., 2007, found a deviation of $\sim 65^{\circ}$ during the T9 flyby), but this would correspond to a very large and unusual deviation.

The analysis of the various pressure terms during T30 shows a different pressure environment, with a much lower total pressure $\left(p_{\text {tot }} \sim 100-300 \mathrm{eV} \mathrm{cm}^{-3}\right.$ ), dominated by the dynamic (with Voyager conditions for the ratio $n_{\mathrm{O}^{+}} / n_{\mathrm{H}^{+}}$, see Sect. 3.2) and magnetic pressure down to at least $1800 \mathrm{~km}$ altitude (not shown here). The ionopause is not clearly defined and depends on the ion temperature (its altitude may be in the range $\sim 1100-1800 \mathrm{~km}$ ), but the thermal pressure is not dominated by the magnetic pressure as during T32. The estimation of the T30 ion temperature using Eq. (1) leads to similar results, with even larger temperature values when the ions are not considered at rest with respect to the neutrals. The ionospheric scale height is apparently larger than during $\mathrm{T} 32$, around $300 \mathrm{~km}$. We then infer an ion exobase at slightly higher altitudes, at about $1600-1700 \mathrm{~km}$ altitude (depending on the ion temperature assumed).

The T30 ionospheric peaks were observed at $\sim 1100 \mathrm{~km}$ altitude (possibly up to $1200 \mathrm{~km}$ ) and $\sim 1250 \mathrm{~km}$ (possibly down to $1050 \mathrm{~km}$ ) respectively in inbound and outbound, with corresponding electron density values around $750 \mathrm{~cm}^{-3}$. The inbound density peak is thus slightly smaller during T30 compared to T32, whereas the outbound peak is much denser during T32 (and at a lower altitude), which may be due to a smaller solar zenith angle. The altitude of the T30 inbound peak is probably slightly lower than during $\mathrm{T} 32$, in agreement with the magnetosheath nightside influence and despite the increased solar zenith angle, but this peak may be broader and extend up to the T32 peak altitude. Globally, a comparison with the statistical analysis of the Titan flybys performed by Ȧgren et al. (2009) reveals that the T32 and T30 ionization peaks characteristics are roughly in agreement with the solar zenith angle dependance observed during those many flybys with Titan in the magnetosphere.

We have completed the data analysis with numerical simulations to study the influence of a magnetosheath plasma on the Titan's nightside ionosphere. The Fig. 4 compares the measured electron densities (discussed previously) in the inbound legs of the T32 and T30 flybys, as well as simulated densities assuming magnetosheath or magnetospheric electrons for the ionization source. We refer to Ágren et 
al. (2007) for the details of the model, which was developed to calculate the electron density profiles in the nightside region and using as input the electron spectra provided by the CAPS-ELS experiment. The model is based on the Rees formula (Rees et al., 1963) and a simplified version of Keller's chemistry scheme (Keller et al., 1998), with an electron transport model (Lummerzheim, 1987) added for the electrons with energies below $200 \mathrm{eV}$. The two CAPS-ELS spectra were taken respectively before and after the T32 encounter ( $16 \mathrm{~h}$ and $18 \mathrm{~h}$, with anode 5), in order to get typical electron spectra in the magnetosphere and the magnetosheath near Titan. The electron flux was decreased by a factor 10 , following the inconsistency observed in the nightside with using the normal flux values (Ägren et al., 2007).

Beyond the quantitative differences between simulated and measured values which are significant above $\sim 1100 \mathrm{~km}$ altitude, we may remark two main features. The first one is the slightly lower simulated densities with a magnetospheric spectrum, due to a smaller electron flux input for electron impact ionization (with a factor of difference of $\sim 2-3$ ). The second one is the different shapes for the simulated ionization peaks: the more energetic magnetospheric electrons create a much broader peak reaching lower altitudes (down to 950$1000 \mathrm{~km}$ altitude, compared to $1150-1250 \mathrm{~km}$ for a magnetosheath spectrum), and the magnetosheath case reveals a second peak around $950 \mathrm{~km}$ with much smaller density values.

If we neglect the quantitative aspect and only consider the altitude profiles, it seems that both $\mathrm{T} 30$ and $\mathrm{T} 32$ electron nightside ionospheric profiles are consistent with a magnetospheric configuration, with no strong decrease in the 950$1200 \mathrm{~km}$ altitude range. However, it is difficult to conclude, since the altitude variability in the density measurements is large, as well as the discrepancies between measurements and simulations, and the CAPS-ELS spectrum during T30 was different from the T32 magnetospheric spectrum. Moreover, the spacecraft was, at low altitudes, near the northern pole where the solar photons may begin to play a significant influence in the ionospheric electrons production. Overall, among the three main differences expected for magnetosheath or magnetospheric ionizing electrons, only the ram direction specificity is clearly present, inducing very different density values in the extended nightside ionosphere. This is consistent with timescales governing the collisionnal ionosphere larger than in the exo-ionosphere, and also larger than the time spent by Titan in the magnetosheath before the observations.

\section{Ionospheric conductivities}

The knowledge of both the electron density (from the Langmuir probe) and the magnetic field (from MAG experiment) allowed us to calculate the ionospheric electrical conductivities during the T32 and T30 Titan flybys. These conductiv-

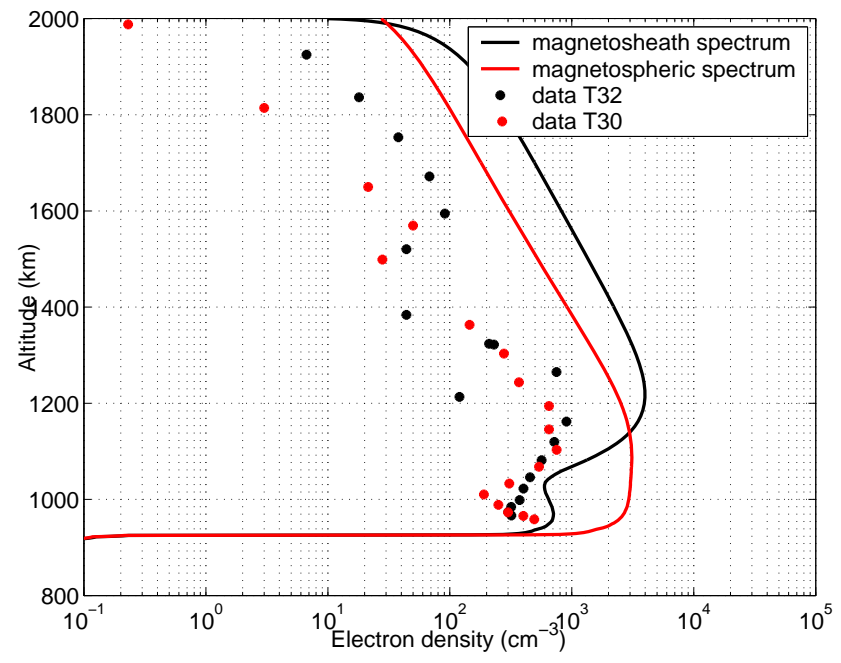

Fig. 4. Electron density in Titan's ionosphere as a function of the altitude $(\mathrm{km})$, measured during the inbound legs of the T32 (black dots) and T30 (red dots) flybys, and modeled using magnetosheath (black continuous line) and magnetospheric (red continuous line) electron spectra.

ities characterize the electrodynamical coupling between the Titan's ionized atmosphere and the incident plasma and magnetic field. We refer to Rosenqvist et al. (2009) for a detailed statistical analysis performed for 17 flybys.

The Pedersen $\left(\sigma_{P}\right)$, Hall $\left(\sigma_{h}\right)$ and parallel $\left(\sigma_{\|}\right)$conductivities, neglecting the ion-electron interactions (negligible up to $2000 \mathrm{~km}$ altitude) are given by (Boström, 1964):

$\left\{\begin{array}{l}\sigma_{P}=\frac{n_{e} e}{|B|}\left(\frac{\Omega_{i} v_{i}}{\Omega_{i}^{2}+v_{i}^{2}}+\frac{\Omega_{e} v_{e}}{\Omega_{e}^{2}+v_{e}^{2}}\right) \\ \sigma_{h}=\frac{n_{e} e}{|B|}\left(\frac{\Omega_{e}^{2}}{\Omega_{e}^{2}+v_{e}^{2}}-\frac{\Omega_{i}^{2}}{\Omega_{i}^{2}+v_{i}^{2}}\right) \\ \sigma_{\|}=n_{e} e^{2}\left(\frac{1}{m_{i} v_{i}}+\frac{1}{m_{e} v_{e}}\right)\end{array}\right.$

with $e$ the electron charge, $\Omega_{i} / \Omega_{e}=\frac{e B}{m_{i} / m_{e}}$ the ion/electron gyrofrequencies (assuming $28 \mathrm{amu} \mathrm{HCNH}^{+}$ions), and $v_{i} / v_{e}$ the ion-neutral/electron-neutral collision frequencies (Kelley et al., 1989).

An important aspect of the ionospheric electrodynamics is the location of the so-called dynamo region. In this region, where the ions are decoupled from the magnetic field lines due to collisions with neutrals $\left(\Omega_{i}<v_{i}\right)$, while the electrons are not $\left(\Omega_{e}>v_{e}\right)$, currents perpendicular to the magnetic field flow and heat the ionosphere. This region is shown in Fig. 5 for the T30 and T32 flybys. The gyrofrequencies are smaller during T30, due to a larger magnetic field, whereas the collisions frequencies are almost unchanged (the figure exhibits only the T32 values). Thus, the dynamo region is roughly between 950 and 1300/1350 km altitude, the lower boundary being only estimated since the closest approach occurred at too high altitudes. For comparison, the Earth's 


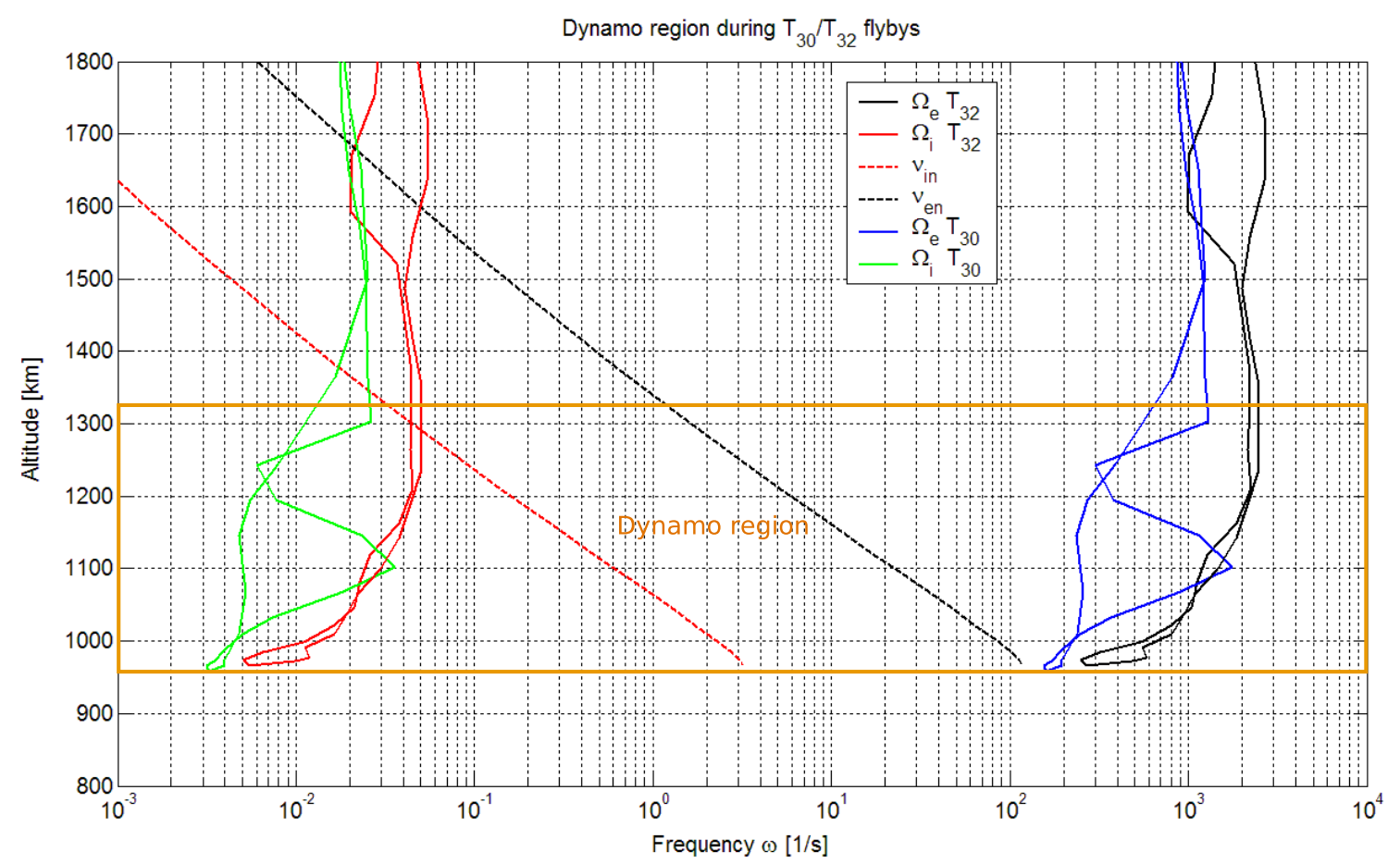

Fig. 5. Dynamo region during the $\mathrm{T} 30$ and $\mathrm{T} 32$ encounters, where the ions are decoupled from the magnetic field, but not the electrons.

mid-latitude dynamo region is located in the $75-130 \mathrm{~km}$ altitude range (Risbeth and Garriott, 1969), and the martian dynamo region is at $\sim 140-205 \mathrm{~km}$ altitude (Withers, 2008). The upper boundaries of the Earth's and Titan's dynamo regions correspond respectively to $2 \%$ and $50 \%$ of their radii. Comparatively, the Titan's dynamo region is much broader and at much higher altitudes, which is related to the larger size of its atmosphere and thus its ionosphere, themselves allowed by a low gravitational binding energy (Johnson, 2004).

The Hall, Pedersen and parallel conductivities are given in Fig. 6 as a function of altitude, for both T30 and T32 flybys (inbound and outbound legs). The comparison between the profiles reveals at first roughly stable profiles, similar to Earth profiles. However, they also reveal an extremely conductive ionosphere: Hall and Pedersen peak values $\left(\sim 8 \times 10^{-2}\right.$ and $\left.4 \times 10^{-2} \mathrm{~S} / \mathrm{m}\right)$ are at least an order of magnitude larger than extreme Earth values observed during geomagnetic disturbances (Rosenqvist et al., 2005).

Several interesting features are observed in the conductivities profiles. First, the values are always larger in the outbound leg than in the inbound one (below the exobase), which is consistent with larger conductivities expected on the dayside ionosphere (induced by larger electron densities). Then, the variability observed is related to the dynamics of the magnetic field, but also to the highly variable electron density, in particular at $\sim 1200 \mathrm{~km}$ where a plasma cavity (see next section) induces a conductivity gap.
The shape of the profiles is very similar to the statistical results obtained by Rosenqvist et al. (2009) during numerous magnetospheric Titan encounters. In particular, we also observe here two Pedersen conductivity peaks: one at the upper boundary of the dynamo region, corresponding to the lower Earth's F-layer due to the electron density peak, and a second one (with even larger values) at the lower boundary of the same region, induced by the brutal magnetic field decrease in the very dense and collision dominated atmosphere. This second peak may be responsible for strong ionospheric electrodynamic processes at altitudes significantly lower than the ionization peak (the second peak altitude is actually unknown, at least below the Cassini closest altitude).

Furthermore, the larger perpendicular conductivities (Hall and Pedersen) as well as the slightly broader dynamo region during T30 compared to T32, may be related to the larger ion ram velocities inferred during T30 with respect to the spacecraft velocity, thus suggesting ion accelerations induced by the electrodynamic coupling between Titan's ionosphere and the ambient plasma and magnetic field. However, some large ram velocity points $(\sim 3-4 \mathrm{~km} / \mathrm{s})$ are probably unrealistic, since they involve an electric field similar to or larger than the corotational electric field.

The knowledge of electrical conductivities during the T32 encounter may help to understand the observation of fossil field lines reported by Bertucci et al. (2008), through the estimation of the magnetic diffusion process. The lifetime of these fossil field lines (with a magnetospheric configuration) 

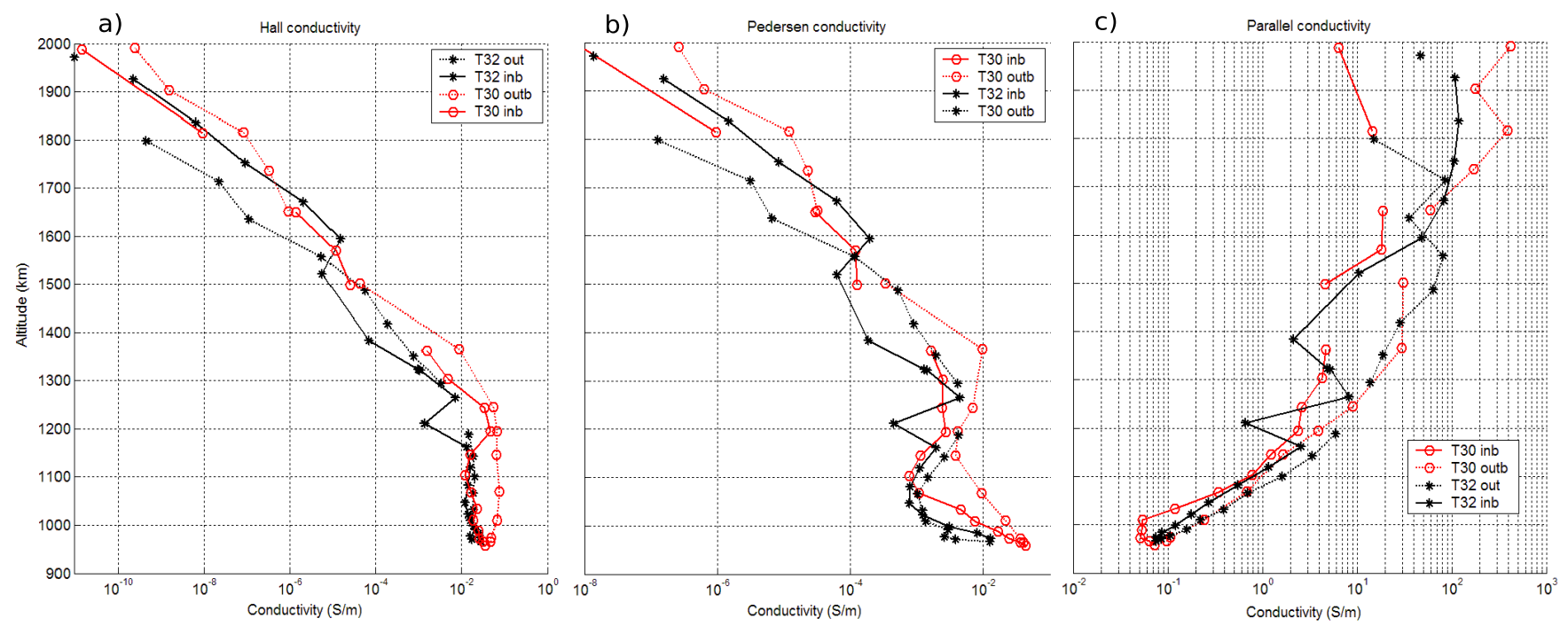

Fig. 6. Electrical conductivities $(\mathrm{S} / \mathrm{m})$ as a function of altitude $(\mathrm{km})$ during the inbound (continuous lines) and outbound (dotted) of the T30 (red) and T32 (black) flybys: Hall (a), Pedersen (b) and parallel conductivities (c).

was estimated at low altitudes between $20 \mathrm{~min}$ and $3 \mathrm{~h}$. The fossil field lines may be replaced by new ones either through diffusion in the collisionnal ionosphere, or through convection towards the downstream region, but also eventually through a reconnection process.

The magnetic field diffusion (Shinagawa et al., 2007) timescale $\tau$ can be estimated knowing the conductivities, via the magnetic diffusion coefficient in the collisionnal ionosphere $D_{m}$ :

$$
\left\{\begin{array}{l}
D_{m}=\frac{1}{\sigma_{\|} \mu_{0}} \\
D_{m}=\frac{l_{m}^{2}}{6 \tau} \\
\frac{1}{l_{m}}=\frac{1}{B}\left|\frac{\partial B}{\partial z}\right|
\end{array}\right.
$$

which leads to ( $z$ being the altitude): $\tau=\frac{1}{6} \sigma_{\|} \mu_{0}\left(\frac{\partial B}{\partial z}\right)^{2}$ The resulting timescale calculated during $\mathrm{T} 32$ increases very rapidly with altitude, from a few minutes only around the closest approach up to several days near the ionization peak. The corresponding diffusion velocity is only a few $\mathrm{km} / \mathrm{s}$ near closest approach, and then rapidly decreases: thus, the electrodynamic processes probably lead to larger convection velocities above $1000 \mathrm{~km}$ altitude (see panel d in Fig. 2). These rough estimates lead to a limit between convection and diffusion dominated regions (regarding the magnetic field) around $1000 \mathrm{~km}$ altitude $( \pm 100 \mathrm{~km})$, with a diffusion timescale around this limit consistent with the $20 \mathrm{~min}-3 \mathrm{~h}$ lifetime inferred by Bertucci et al. (2008) for fossil field lines. The replacement of such field lines observed at much higher altitudes probably rather involves either convection or reconnection mechanisms. We may add that Ma et al. (2009) and Simon et al. (2009) showed the importance of reconnection processes in the reconfiguration of the magnetotail plasma during the T32 flyby.

A more precise analysis would be needed to investigate the dynamics of the magnetic field in the lower ionosphere, through the magnetic diffusion/convection approach, as developed by Keller et al. (1994) before the Cassini mission with a one-dimensional magnetohydrodynamical model of Titan's ionosphere. We may add that our values for the magnetic diffusion coefficient are very similar to those calculated by Keller et al. (1994) near 1000-1200 km altitude when considering the correct solar zenith angles, with for example $D_{m} \sim 10^{9}$ and $1.5 \times 10^{9} \mathrm{~cm}^{2} \mathrm{~s}^{-1}$ respectively for our results and those shown by Keller et al. (1994).

\section{A polar plasma cavity}

The detailed analysis of the ionospheric electron density given by the Langmuir probe reveals the existence of a polar plasma cavity during the T32 Titan flyby. This strong depletion appears at $\sim 1200 \mathrm{~km}$ altitude in the inbound leg of the encounter (see the blue zone in Fig. 2).

The comparison with the outbound leg of the same encounter or with other deep flybys (such as T30) confirms that the structure observed corresponds to a strong depletion, and not to "normal" density values surrounded by strong density peaks. The electron density inferred by the probe is roughly decreased by a factor 10 in this cavity. The apparent width (assuming a structure at rest) is of about $20-30 \mathrm{~km}$ and the spacecraft was located above the northern pole at a solar zenith angle near $\sim 130^{\circ}$ and a Titan latitude around $60-65^{\circ}$.

We might argue a technical problem with the Langmuir probe, since there is only one sweep point available, with a 


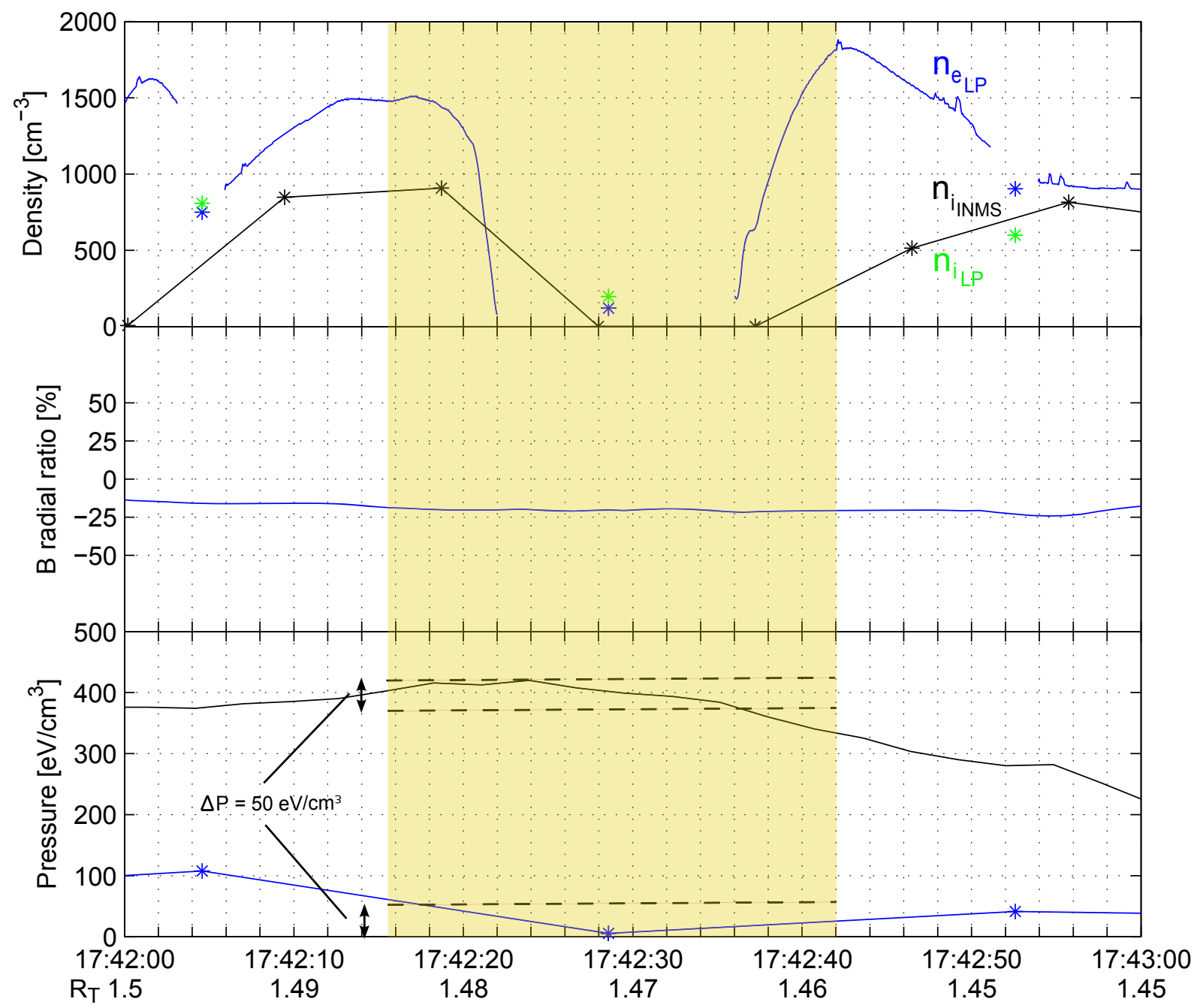

Fig. 7. Analysis of Cassini data (versus time) around the period of the plasma cavity found in the inbound part of the T32 flyby. The distance from Titan (in $R_{T}$ ) is given along the time axis. The first panel gives: the electron density $n_{e} L$ given by the Langmuir probe (sweep data with stars, and $20 \mathrm{~Hz}$ density in continuous lines), the ion density given by the Langmuir probe ( $n_{i_{L P}}$, green) and the INMS instrument ( $n_{i_{\mathrm{INMS}}}$, black). The second panel gives the ratio (in \%) $B_{r} / B$, with $B_{r}$ and $B$ being respectively the radial component and the total value of the local magnetic field measured by MAG. The last panel gives the thermal (blue) and magnetic (black) pressures.

fit of the current-voltage curve inducing larger uncertainties than usual in the ionosphere. However, other instruments detected the same strong depletion.

The Fig. 7 shows indeed a zoom of this depletion region, with the upper panel giving the Langmuir probe electron $\left(n_{e_{L P}}\right)$ and ion $\left(n_{i_{L P}}\right)$ densities, as well as the ion densities derived by the INMS experiment $\left(n_{i_{\mathrm{INMS}}}\right)$. All profiles show a strong plasma depletion in the same time. Furthermore, the electron density derived from the RPWS upper hybrid emissions is also very significantly decreased (by a factor $3-4$, see Fig. 2).

In addition, a zoom of the thermal and magnetic pressures (lower panel in Fig. 7) reveals a pressure balance between them, with a reduced thermal pressure counterbalanced by a magnetic pressure increase of the same order. Thus, this pressure balance allows the existence of such a cavity, even if it does not explain its formation process.

Is this depletion a permanent feature? The density profiles in the ionosphere are often highly variable, but this depletion is the strongest observed today. However, an analysis of the previous low altitude encounters reveals strong and localized depletions. The flybys T5 (16 April 2005), T21 (12 December 2006) and possibly T26 (10 March 2007) and T30 (12 May 2007) exhibit such features. The altitudes concerned are variable, but all of them are detected above the northern pole at solar zenith angle values near $130-140^{\circ}$ or latitudes around $60-70^{\circ}$ and Titan local times of $22-02 \mathrm{~h}$. These results seem in agreement with the existence of a plasma cavity 
near the northern pole. Nonetheless, not all the flybys visiting the same region exhibit a strong cavity, even if the altitude ranges concerned for the spacecraft may be different from the corresponding altitude of the cavity.

Three main types of plasma cavities, involving either chemical loss or field-aligned plasma accelerations, may be considered to study the source mechanisms for the features observed in Titan's ionosphere: the Earth's auroral cavities, the nightside Venus holes, and the depletions artificially induced by exhaust gases.

The Earth's auroral cavities correspond to localized fieldaligned plasma depletions in the auroral region, reduced down to $70 \%$ of the surrounding density values (Doe et al., 1993), characterized by parallel currents accelerating magnetospheric electrons downward and ionospheric ions upward. Ionospheric holes were also reported and analyzed in the case of Venus (see Brace et al., 1991, or Luhmann et al., 1982): these are large-scale vertical structures in the nightside region, with strong radial magnetic fields preventing horizontal transport of the plasma, filled with both ionospheric and magnetotail plasma populations, and located symmetrically at low latitudes in the wake (by pairs).

The Cassini MAG observations reveal a strong rotation of the magnetic field near the observed cavity, which is however related to the existence of fossil field lines in the deep ionosphere of Titan (Bertucci et al., 2008). Actually, the radial component of the magnetic field is rather stable and small around the observed cavity (see Fig. 7 middle panel), which is in contradiction with formation processes like the Earth's auroral cavities or the nightside Venus holes where the magnetic field is mostly vertical with a strong rotation at the edges of the structure.

Moreover, the CAPS observations show no clear acceleration of electrons or ions at the same period, even if the orientation of the anodes with respect to the magnetic field was not suited to properly reveal field-aligned beams. We may add that the RPWS (waves experiment) frequency range above $1 \mathrm{~Hz}$ - does not allow to observe Alfven waves such as those observed at the edges of the Earth's auroral cavities, due to a low magnetic field and large ionospheric densities.

We may mention that the ion ram velocity inferred by the Langmuir probe inside the cavity is significantly increased $\left(\sim 10 \mathrm{~km} / \mathrm{s}\right.$ to be compared with $\left.v_{s c}=6 \mathrm{~km} / \mathrm{s}\right)$ and that the deduced averaged ion mass is decreased by a factor 4 in the same time: this would suggest the presence of light species ion flows. However, both parameters are calculated assuming a charge neutrality $\left(n_{i}=n_{e}\right)$, so that the large uncertainties on the electron density in the cavity lead to uncertain values for $v_{i, \text { ram }}$ and $m_{i}$.

Chemical loss is an other candidate mechanism for the formation of plasma cavities. In particular, plasma cavities observed in the Earth's ionosphere were artificially induced by the exhaust gases of the spacecraft, such as at the time of the Skylab launch in 1973 (Mendillo et al., 1975; Mendillo, 1988). The released $\mathrm{H}_{2} \mathrm{O}$ molecules reacted very efficiently with the atomic ions of the ionosphere $\left(\mathrm{H}^{+}\right.$or $\left.\mathrm{O}^{+}\right)$, leading to a local production of molecular ions $\left(\mathrm{H}_{2} \mathrm{O}^{+}\right.$or $\left.\mathrm{H}_{3} \mathrm{O}^{+}\right)$ whose electron recombination rates are much larger (a factor 1000) than those of atomic ions. Thus, a local plasma cavity was artificially created within a few minutes and lasted one or two hours. Moore and Mendillo (2007) suggested that similar mechanisms are also active in the Saturn's ionosphere. However, Titan's ionosphere is most probably not subject to such processes. First, the major ionospheric ions are already molecular - i.e. $\mathrm{HCNH}^{+} / \mathrm{C}_{2} \mathrm{H}_{5}^{+} / \mathrm{CH}_{5}^{+}$(Cravens et al., 2006) - , so that their recombination rates with electrons are high and of the same order as for $\mathrm{H}_{2} \mathrm{O}^{+}$or $\mathrm{H}_{3} \mathrm{O}^{+}$(Anicich and McEwan, 1997). Moreover, given the time for the formation and the disappearing of artificial cavities, the Cassini spacecraft could neither detect them just after their creation nor during the next flyby.

One may finally note that the CAPS-ELS instrument detected negative ions just after (or below) the polar cavity (Coates et al., 2007b). This is most probably a coincidence, but it might suggest that chemical or electrodynamical interactions involving these species lead to density depletions at the transition layer with the negative ions region.

As a consequence, the formation of the cavity observed during T32 and of the other depletions detected in the same region during other flybys is not well understood, which is in particular due to the absence of 3-D distributions for the ions and electrons. The numerous Titan flybys will hopefully allow in the future to better establish the permanence of this cavity, and eventually give a better insight into its formation process with the use of multi-instruments datasets.

\section{Summary}

The paper is dedicated to the analysis of the Langmuir probe data during the T32 Titan flyby. This encounter, which occurred on 13 June 2007, is the only one where Titan was outside the magnetosphere, in the magnetosheath. This gave the possibility, for the first time, to study Titan's ionosphere with a magnetosheath plasma environment.

We first analyze the plasma environment outside the dense ionosphere (Sect. 3), combining the Langmuir probe results with CAPS data, revealing an asymmetric environment and the presence, after the magnetopause crossing, of an extended cold exo-ionospheric region in the inbound leg of the flyby. This dense region is most probably induced by the direction of the magnetosheath plasma dragging the cold plasma downstream. The analysis of all pressure terms magnetic, energetic, thermic and dynamic - completes the picture of the plasma environment, showing a magnetic pileup near Titan which may act as a barrier for the incident electrons (thus playing a role concerning the actual discrepancies between observations and modeling in the nightside ionosphere). 
The ionosphere is then studied in detail (Sect. 4), with an expected asymmetry between inbound and outbound legs in agreement with a mostly solar-driven ionosphere. The T32 ionopause was located at low altitudes (around $\sim 1000 \mathrm{~km}$ altitude only) and large ion flows may be observed in the deep ionosphere. The ion temperature is derived, either assuming the ions at rest with respect to the neutrals or considering the ram velocities inferred by the Langmuir probe. This leads to an estimated ion exobase altitude about 100 or $200 \mathrm{~km}$ above the neutral exobase.

A comparison between the T32 and T30 flybys is given (Sect. 5), since the geometries of the encounters with respect to both Saturn and Titan are very similar, but Titan was inside the magnetosphere during T30. This provides a reference case to study the influence of a magnetosheath incident plasma on the nightside ionosphere, where the electron impact ionization process is the main source for the ionosphere. The combination of Langmuir probe data and modeled electron densities (using magnetosheath or magnetospheric spectra as input) reveals that the nightside ionization peak altitude may be influenced during T32 by the magnetosheath plasma (a lower electron temperature leading to a higher peak altitude), but no significant difference in the ionospheric densities was inferred in the nightside ionosphere between both flybys, despite a larger incident electron flux in the magnetosheath. The most important influence is the change of the ionospheric shape induced by the different incident plasma direction, with an extended ionospheric tail observed during T32 and not T30.

The electrical conductivities (Hall, Pedersen and parallel) are calculated in order to characterize the electrodynamical coupling between Titan's ionosphere and the incident plasma and magnetic field. The dynamo region, where currents perpendicular to the magnetic field flow, is located between 950 (or below) and 1300/1350 km altitude, which makes it a very broad (and at high altitudes) dynamo region compared to the Earth. The conductivities reveal a highly conductive ionosphere, with two conductivity peaks observed. The comparison between the ion ram velocities and the conductivities during the T32 and T30 flybys suggests ion flows induced by the electrodynamical coupling between the ionosphere and the ambient magnetic field. Furthermore, the calculation of the magnetic field diffusion timescale in the ionosphere is in agreement with the lifetime of fossil magnetic field lines near closest approach obtained by Bertucci et al. (2008) during T32, but shows the importance of either convection or reconnection for the replacement of these fossil field lines at higher altitudes.

A polar plasma cavity is observed during T32 above the northern pole, at latitudes around $60-65^{\circ}$ in the inbound leg, with a large electron and ion density depletion derived from the Langmuir probe, the RPWS upper hybrid frequency and the INMS experiment. Moreover, similar density depletions are observed during several Titan flybys, systematically in the same region above the northern pole. The cavity is made possible by a pressure balance between the magnetic and thermal pressures, but neither the Earth's auroral cavities, nor the Venus nightside holes nor the cavities artificially induced by spacecraft's exhaust gases are processes which seem appropriate to explain its formation. However, the absence of 3-D plasma distributions combined with the unappropriate orientation of the plasma spectrometers (with respect to the magnetic field direction) are major constraints for the analysis. The detailed understanding of the cavity formation mechanisms will need further study in the future.

Acknowledgements. We would like to thank M. Morooka, P.L. Blelly, D. Toublanc, I. Dandouras and A. Opitz for useful discussions, as well as the colleagues in IRFU who participated in building the Langmuir probe or storing the data.

Topical Editor M. Pinnock thanks S. Simon, T. Cravens, and another anonymous referee for their help in evaluating this paper.

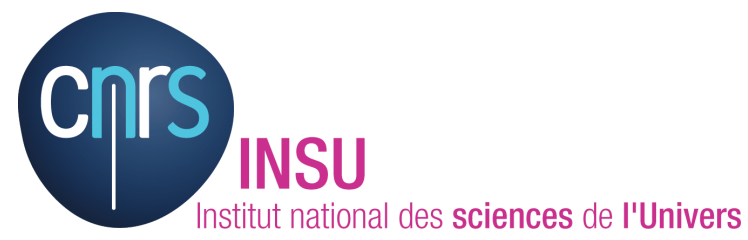

The publication of this article is financed by CNRS-INSU.

\section{References}

Achilleos, N., Arridge, C. S., Bertucci, C., Jackman, C. M., Dougherty, M. K., Khurana, K. K., and Russell, C. T.: Large-scale dynamics of Saturns magnetopause: Observations by Cassini, J. Geophys. Res., 113, A11209, doi:10.1029/2008JA013265, 2008.

Ȧgren, K., Wahlund, J.-E., Modolo, R., Lummerzheim, D., Galand, M., Müller-Wodarg, I., Canu, P., Kurth, W. S., Cravens, T. E., Yelle, R. V., J. H. Waite Jr., Coates, A. J., Lewis, G. R., Young, D. T., Bertucci, C., and Dougherty, M. K.: On magnetospheric electron impact ionisation and dynamics in Titan's ram-side and polar ionosphere - a Cassini case study, Ann. Geophys., 25, 23592369, 2007,

http://www.ann-geophys.net/25/2359/2007/.

Ȧgren, K., Wahlund, J.-E., Garnier, P., Modolo, R., Cui, J., Galand, M., and Müller-Wodarg, I.: On the ionospheric structure of Titan, Planet. Space Sci., in press, doi:10.1016/j.pss.2009.04.012, 2009.

Anicich, V. G. and McEwan, M. J.: Ion-molecule chemistry in Titan's ionosphere, Planet. Space Sci., 45, 897-921, 1997.

Backes, H., Neubauer, F. M., Dougherty, M. K., Achilleos, N., André, N., Arridge, C. S., Bertucci, C., Jones, G. H., Khurana, K. K., Russell, C. T., and Wennmacher, A.: Titan's magnetic field signature during the first Cassini encounter, Science, 308, 992-995, 2005.

Banks, P. M. and Kockarts, G.: Aeronomy, part A, Academic Press, 1979

Blelly, P.-L. and Alcaydé, D.: Ionosphere, Handbook of the SolarTerrestrial Environment, edited by: Kamide, Y. and Chian, A. C., p. $190,2007$. 
Bertucci, C., Achilleos, N., Dougherty, M. K., Modolo, R., Coates, A. J., Szego, K., Masters, A., Ma, Y., Neubauer, F. M., Garnier, P., Wahlund, J.-E., and Young, D. T.: The Magnetic Memory of Titan's Ionized Atmosphere, Science, 321, 1474-1478, 2008.

Boström, R.: A Model of the Auroral Electrojects, J. Geophys. Res. 69, 4983-4999, 1964.

Brace, L. H. and Kliore, A. J.: The structure of the Venus ionosphere, Space Sci. Rev., 55, 81-163, 1991.

Bridge, H. S., Bagenal, F., Belcher, J. W., Lazarus, A. J., McNutt, R. L., Sullivan, J. D., Gazis, P. R., Hartle, R. E., Ogilvie, K. W., Scudder, J. D., Sittler, E. C., Eviatar, A., Siscoe, G. L., Goertz, C. K., and Vasyliunas, V. M.: Plasma Observations Near Saturn: Initial Results from Voyager 2, Science, 215, 563-570, 1982.

Coates, A. J., Crary, F. J., Lewis, G. R., Young, D. T., Waite, J. H., and Sittler, E. C.: Discovery of heavy negative ions in Titan's ionosphere, Geophys. Res. Lett., 34, L22103, doi:10.1029/2007GL030978, 2007.

Coates, A. J., McAndrews, H. J., Arridge, C. S., Jones, G. H., Crary, F. J., Young, D. T., Szego, K., Sittler, E. C., Thomsen, M. F., Tokar, R. L., Bertucci, C., and Dougherty, M. K.: Titan at Saturn's magnetopause: CAPS results from T32, American Geophysical Union, Fall Meeting, 2007.

Cravens, T. E., Robertson, I. P., Waite, J. H., Yelle, R. V., Kasprzak, W. T., Keller, C. N., Ledvina, S. A., Niemann, H. B., Luhmann, J. G., McNutt, R. L., Ip, W.-H., De La Haye, V., Mueller-Wodarg, I., Wahlund, J.-E., Anicich, V. G., and Vuitton, V.: Composition of Titan's ionosphere, Geophys. Res. Lett., 33, L07105, doi:10.1029/2005GL025575, 2006.

Cravens, T. E., Robertson, I. P., Waite, J. H., Yelle, R. V., Vuitton, V., Coates, A. J., Wahlund, J.-E., Agren, K., Richard, M. S., de La Haye, V., Wellbrock, A., and Neubauer, F. M.: Model-data comparisons for Titan's nightside ionosphere, Icarus, 199, 174188, 2009.

Doe, R. A., Mendillo, M., Vickrey, J. F., Zanetti, L. J., Eastes, R. W., Observations of Nightside Auroral Cavities, J. Geophys. Res., 98, 293-310, 1993.

Dougherty, M. K., Kellock, S., Southwood, D. J., Balogh, A., Smith, E. J., Tsurutani, B. T., Gerlach, B., Glassmeier, K.-H., Gleim, F., Russell, C. T., Erdos, G., Neubauer, F. M., and Cowley, S. W. H.: The Cassini Magnetic Field Investigation, Space Sci. Rev., 114(1-4), 331-383, 2004.

Fahleson, U., Fälthammar, C.-G., and Pedersen, A.: Ionospheric temperature and density measurements by means of spherical double probes, Planet. Space Sci., 22, 41-66, 1974.

Galand, M., Yelle, R. V., Coates, A. J., Backes, H., and Wahlund, J.E.: Electron temperature of Titan's sunlit ionosphere, Geophys. Res. Lett., 33, L21101, doi:10.1029/2006GL027488, 2006.

Gan, L., Keller, C. N., and Cravens, T. E.: Electrons in the ionosphere of Titan, J. Geophys. Res., 97, 12137-12151, 1992.

Garnier, P., Dandouras, I., Toublanc, D., Brandt, P. C., Roelof, E. C., Mitchell, D. G., Krimigis, S. M., Krupp, N., Hamilton, D. C., and Waite, H.: The exosphere of Titan and its interaction with the kronian magnetosphere: MIMI observations and modeling, Planet. Space Sci., 55, 165-173, 2007.

Gurnett, D. A., Kurth, W. S., Kirchner, D. L., Hospodarsky, G. B., Averkamp, T. F., Zarka, P., Lecacheux, A., Manning, R., Roux, A., Canu, P., Cornilleau-Wehrlin, N., Galopeau, P., Meyer, A., Boström, R., Gustafsson, G., Wahlund, J.-E., Ahlen, L., Rucker, H. O., Ladreiter, H. P., Macher, W., Woolliscroft, L. J. C., Al- leyne, H., Kaiser, M. L., Desch, M. D., Farrell, W. M., Harvey, C. C., Louarn, P., Kellogg, P. J., Goetz, K., and Pedersen, A.: The Radio and Plasma Wave Science Investigation, Space Sci. Rev., 114, 395-463, 2004.

Hartle, R. E, Sittler, E. C., Neubauer, F. M., Johnson, R. E., Smith, H. T., Crary, F., McComas, D. J., Young, D. T., Coates, A. J., Simpson, D., Bolton, S., Reisenfeld, D., Szego, K., Berthelier, J. J., Rymer, A., Vilppola, J., Steinberg, J. T., and Andre, N.: Initial interpretation of Titan plasma interaction as observed by the Cassini plasma spectrometer: Comparisons with Voyager 1, Planet. Space Sci., 54, 1211-1224, 2006 .

Hartle, R. E., Sittler, E. C., Neubauer, F. M., Johnson, R. E., Smith, H. T., Crary, F., McComas, D. J., Young, D. T., Coates, A. J., Simpson, D., Bolton, S., Reisenfeld, D., Szego, K., Berthelier, J. J., Rymer, A., Vilppola, J., Steinberg, J. T., and Andre, N.: Preliminary interpretation of Titan plasma interaction as observed by the Cassini Plasma Spectrometer: Comparisons with Voyager 1, Geophys. Res. Let., 33, L08201, doi:10.1029/2005GL024817, 2006b.

Johnson, R. E.: The magnetospheric plasmadriven evolution of satellite atmospheres, The Astrophys. J., 609, 99-102, 2004.

Keller, C. N., Cravens, T. E., and Gan, L.: One dimensional multispecies magnetohydrodynamic models of the ramside ionosphere of Titan, J. Geophys. Res., 99, 6511-6525, 1994.

Keller, C. N., Anicich, V. G., and Cravens, T. E.: Model of Titan's ionosphere with detailed hydrocarbon ion chemistry, Planet. Space Sci., 46, 1157-1174, 1998.

Kelley, M. C.: The Earth's ionosphere, Plasma Physics and Electrodynamics, vol. 43, Academic Press, London, UK, 1989.

Krimigis, S. M., Mitchell, D. G., Hamilton, D. C., Livi, S., Dandouras, J., Jaskulek, S., Armstrong, T. P., Boldt, J. D., Cheng, A. F., Gloeckler, G., Hayes, J. R., Hsieh, K. C., Ip, W.-H., Keath, E. P., Kirsch, E., Krupp, N., Lanzerotti, L. J., Lundgren, R., Mauk, B. H., McEntire, R. W., Roelof, E. C., Schlemm, C. E., Tossman, B. E., Wilken, B., and Williams, D. J.: Magnetosphere imaging instrument (MIMI) on the Cassini Mission to Saturn/Titan, Space Sci. Rev., 114, 233-329, 2004.

Lemaire, J. and Scherer, M.: Exospheric models of the topside ionosphere, Space Sci. Rev., 15, 591-640, 1974.

Luhmann, J. G., Russell, C. T., Brace, L. H., Knudsen, W. C., Taylor, H. A., Scarf, F. L., Colburn, D. S., and Barnes, A.: Pioneer Venus Observations of Plasma and Field Structure in the Near Wake of Venus, J. Geophys. Res., 87, 9205-9210, 1982.

Lummerzheim, D.: Electron Transport and Optical Emissions in the Aurora, PhD Thesis, University of Alaska, Fairbanks, 1987.

Lundberg, E. T., Hansen, K. C., Gombosi, T. I., and Toth, G.: Statistical study of the probability of Titan being in the solar wind or in Saturn's magnetosheath, AGU fall meeting, 2005.

Ma, Y. J., Russell, C. T., Nagy, A. F., Toth, G., Bertucci, C., Dougherty, M. K., Neubauer, F. M., Wellbrock, A., Coates, A. J., Garnier, P., Wahlund, J.-E., Cravens, T. E., and Crary, F. J.: Time-dependent global MHD simulations of Cassini T32 flyby: from magnetosphere to magnetosheath, J. Geophys. Res., 114, A03204, doi:10.1029/2008JA013676, 2009.

Mendillo, M., Hawkins, G. S., and Klobuchar, J. A.: A Sudden Vanishing of the Ionospheric F Region Due to the Launch of Skylab, J. Geophys. Res., 80, 2217-2228, 1975.

Mendillo, M.: Ionospheric holes: a review of theory and recent experiments, Adv. Space Res., 8, 151-162, 1988. 
Modolo, R., Wahlund, J.-E., Boström, R., Canu, P., Kurth, W. S., Gurnett, D., Lewis, G. R., and Coates, A. J.: Far plasma wake of Titan from the RPWS observations: A case study, Geophys. Res. Lett., 34, L24S04, doi:10.1029/2007GL030482, 2007.

Modolo, R., Chanteur, G. M., Wahlund, J.-E., Canu, P., Kurth, W. S., Gurnett, D., Matthews, A. P., and Bertucci, C.: Plasma environment in the wake of Titan from hybrid simulation: A case study, Geophys. Res. Lett., 34, L24S07, doi:10.1029/2007GL030489, 2007.

Moore, L. and Mendillo, M.: Are plasma depletions in Saturns ionosphere a signature of time dependent water input?, Geophys. Res. Lett., 34, L12202, doi:10.1029/2007GL029381, 2007.

Mott-Smith, H. M. and Langmuir, I.: The theory of collectors in gaseous discharges, Phys. Rev., 28, 727-763, 1926.

Müller-Wodarg, I. C. F., Yelle, R. V., Borggren, N., and Waite, J. H.: Waves and horizontal structures in Titan's thermosphere, J. Geophys. Res., 113, A12315, doi:10.1029/2006JA011961, 2006.

Müller-Wodarg, I. C. F, Yelle, R. V., Cui, J., and Waite, J. H.: Horizontal structures and dynamics of Titan's thermosphere, J. Geophys. Res., 113, E10005, doi:10.1029/2007JE003033, 2008.

Neubauer, F. M., Gurnett, D. A., Scudder, J. D., and Hartle, R. E.: Titan's magnetospheric interaction, in Saturn, edited by: University of Arizona Press, 760-767, 1984.

Richardson, J. D.: Ion Distributions in the Dayside Magnetosheaths of Jupiter and Saturn, J. Geophys. Res., 92, 6133-6140, 1987.

Risbeth, H. and Garriott, O. K.: Introduction to Ionospheric Physics, Elsevier, New York, 1969.

Roboz, A. and Nagy, A. F.: The energetics of Titan's ionosphere, J. Geophys. Res., 99, 2087-2093, 1994.

Rosenqvist, L., Opgenoorth, H., Buchert, S., McCrea, I., Amm, O., and Lathuillere, C.: Extreme solar-terrestrial events of October 2003: High-latitude and Cluster observations of the large geomagnetic disturbances on 30 October, J. Geophys. Res., 110(A9), A09S23, doi:10.1029/2004JA010927, 2005.

Rosenqvist, L., Wahlund, J.-E., Ȧgren, K., Modolo, R., Opgenoorth, H. J., Strobel, D., Müller-Wodarg, I., Garnier, P., and Bertucci, C.: Titan ionospheric conductivities from Cassini measurements, Planet. Space Sci., in press, doi:10.1016/j.pss.2009.01.007, 2009.

Schunk, R. W. and Nagy, A. F.: Ionospheres: Physics, Plasma Physics, Chemistry, Cambridge University Press, 2000.

Sergis, N., Krimigis, S. M., Mitchell, D. G., Hamilton, D. C., Krupp, N., Mauk, B. H., Roelof, E. C., and Dougherty, M. K.: Ring current at Saturn: Energetic particle pressure in Saturn's equatorial magnetosphere measured with Cassini/MIMI, Geophys. Res. Lett., 34, L09102, doi:10.1029/2006GL029223, 2007.

Sergis, N., Krimigis, S. M., Mitchell, D. G., Hamilton, D. C., Krupp, N., Mauk, B. H., Roelof, E. C., and Dougherty, M. K.: Energetic particle pressure in Saturn's magnetosphere measured with the Magnetospheric Imaging Instrument on Cassini, J. Geophys. Res., 114, A02214, doi:10.1029/2008JA013774, 2009.
Shinagawa, H., Cravens, T. E., and Nagy, A. F.: A one-dimensional time-dependent model of the magnetized ionosphere of Venus, J. Geophys. Res., 92, 7317-7330, 1987.

Simon, S., Motschmann, U., Kleindienst, G., Saur, J., Bertucci, C. L., Dougherty, M. K., Arridge, C. S., and Coates, A. J.: Titan's plasma environment during a magnetosheath excursion: Realtime scenarios for Cassini's T32 flyby from a hybrid simulation, Ann. Geophys., 27, 669-685, 2009, http://www.ann-geophys.net/27/669/2009/.

Szego, K., Bebesi, Z., Bertucci, C., Coates, A. J., Crary, F., Erdos, G., Hartle, R., Sittler, E. C., and Young, D. T.: Charged particle environment of Titan during the T9 flyby, Geophys. Res. Lett., 34, L24S03, doi:10.1029/2007GL030677, 2007.

Rees, M. H.: Physics and chemistry of the upper atmosphere, Cambridge University Press, 1963.

Toublanc, D., Parisot, J. P., Brillet, J., Gautier, D., Raulin, F., and McKay, C. P.: Photochemical modeling of Titan's atmosphere, Icarus, 113, 2-26, 1995.

Vuitton, V., Yelle, R. V., and Anicich, V. G.: The nitrogen chemistry of Titan's upper atmosphere revealed, Astrophys. J., 647, L175L178, 2006.

Wahlund, J.-E., Boström, R., Gustafsson, G., Gurnett, D. A., Kurth, W. S., Pedersen, A., Averkamp, T. F., Hospodarsky, G. B., Persoon, A. M., Canu, P., Neubauer, F. M., Dougherty, M. K., Eriksson, A. I., Morooka, M. W., Gill, R., Andr, M., Eliasson, L., and Müller-Wodarg, I.: Cassini Measurements of Cold Plasma in the Ionosphere of Titan, Science, 308, 986-989, 2005.

Waite, J. H., Lewis, W. S., Kasprzak, W. T., Anicich, V. G., Block, B. P., Cravens, T. E., Fletcher, G. G., Ip, W.-H., Luhmann, J. G., McNutt, R. L., Niemann, H. B., Parejko, J. K., Richards, J. E., Thorpe, R. L., Walter, E. M., and Yelle, R. V.: The Cassini Ion and Neutral Mass Spectrometer (INMS) Investigation, Space Sci. Rev., 114, 113-231, 2004.

Waite, J. H., Niemann, H., Yelle, R. V., Kasprzak, W., Cravens, T., Luhmann, J., McNutt, R., Ip, W.-H., Gell, D., Muller-Wordag, I. C. F., Ledvina, S., Magee, B., Borggren, N., Fletcher, G., Walter, E., Miller, R., Xu, J., Block, B., and Arnett, K.: Ion Neutral Mass Spectrometer results from the first flyby of Titan, Science, 308, 982-986, 2005.

Waite, J. H., Young, D. T., Cravens, T. E., Coates, A. J., Crary, F. J., Magee, B., and Westlake, J.: The Process of Tholin Formation in Titan's Upper Atmosphere, Science, 316, 870-875, 2007.

Withers, P.: Theoretical models of ionospheric electrodynamics and plasma transport, J. Geophys. Res., 113, A07301, doi:10.1029/2007JA012918, 2008. 